УДК 519.21

\title{
Logarithmic Barrier Method Via Minorant Function for Linear Programming
}

\author{
Assma Leulmi* \\ Department of Mathematics, Faculty of Sciences \\ Ferhat Abbas University of Setif-1, 19000 \\ Algeria \\ Soumia Leulmi \\ Department of Mathematics \\ University Mohamed Khider of Biskra \\ Algeria
}

Received 14.10.2018, received in revised form 10.01.2019, accepted 13.02.2019

We propose in this study, a new logarithmic barrier approach to solve linear programming problem. We are interested in computation of the direction by Newton's method and of the displacement step using minorant functions instead of line search methods in order to reduce the computation cost.

Our new approach is even more beneficial than classical line search methods. This purpose is confirmed by many interesting numerical experimentations shown the effectiveness of the algorithm developed in this work.

Keywords: linear programming, logarithmic barrier methods, line search.

DOI: 10.17516/1997-1397-2019-12-2-191-201.

\section{Introduction}

Interior-point methods are one of the efficient methods developed to solve linear and non linear programming problems.

Several algorithms have been proposed to solve the linear programming problem, where, we distinguish three fundamental classes of interior point methods namely: projective interior point methods and their alternatives, central trajectory methods, barrier/penalty methods [2]. Our work is based on the latter type of interior point methods for solving linear programming problems.

In this paper, we propose a logarithmic barrier interior-point method for solving linear programming problems (LP). In fact, the main difficulty to be anticipated in establishing an iteration in such a method will come from the determination and computation of the step-size. Various approaches are developed to overcome this difficulty. It is known $[2,6]$ that the computation of the step-size is expensive specifically while using line search methods. Leulmi and al. [5] proposed efficient and less expensive procedures in semidefinite programming not only to avoid line search methods, but also to accelerate the algorithm's convergence. The purpose of this paper is to exploit this idea for LP problems.

We consider the following linear programming problem

$$
\text { (D) }\left\{\begin{array}{l}
\min _{x} b^{T} x \\
A^{T} x \geqslant c, x \in \mathbb{R}^{m} .
\end{array}\right.
$$

Where $A \in \mathbb{R}^{m \times n}$, such that $\operatorname{rang} A=m<n, c \in \mathbb{R}^{n}$ and $b \in \mathbb{R}^{m}$.

*as_smaleulmi@yahoo.fr

(c) Siberian Federal University. All rights reserved 
The problem $(D)$ is the dual of the following linear program

$$
(P)\left\{\begin{array}{l}
\max _{y} c^{T} y \\
A^{T} y=b, \\
y \in \mathbb{R}^{n}, y \geqslant 0 .
\end{array}\right.
$$

The problem $(D)$ can be written in the following standard form

$$
(D)\left\{\begin{array}{l}
\max _{x} c^{T} x \\
A^{T} x-c=s \\
x \in \mathbb{R}^{m}, s \in \mathbb{R}^{n}, s \geqslant 0 .
\end{array}\right.
$$

A priori, one of the advantages of the problem $(D)$ with respect to its dual problem $(P)$ is that variable of the objectif function is a vector instead to be a matrix in the type problem $(P)$. Furthermore, under certain convenient hypothesis, the resolution of the problem $(D)$ is equivalent of the problem $(P)$ in the sense that the optimal solution of one of the two problems can be reduced directly from the other through the application of the theorem of the sladeness complementary, see for instance [7].

In all which follows, we denot by

1. $X=\left\{x \in \mathbb{R}^{m}: A^{T} x-c \geqslant 0\right\}$, the set of feasible solutions of $(D)$.

2. $\widehat{X}=\left\{x \in \mathbb{R}^{m}: A^{T} x-c>0\right\}$, the set of strictly feasible solutions of $(D)$.

3. $F=\left\{y \in \mathbb{R}^{n}: A y=b, y \geqslant 0\right\}$, the set of feasible solutions of $(P)$.

4. $\widehat{F}=\left\{y \in \mathbb{R}^{n}: A y=b, y>0\right\}$, the set of strictly feasible solutions of $(P)$.

Let $u, v \in \mathbb{R}^{n}$, their scalar product is defined by

$$
\langle u, v\rangle=u^{T} v=\sum_{i=1}^{n} u_{i} v_{i}
$$

We suppose that the sets $\widehat{X}$ and $\widehat{F}$ are not empty.

The problem $(D)$ is approximated by the following perturbed problem $\left(D_{\eta}\right)$

$$
\left(D_{\eta}\right)\left\{\min f_{\eta}(x): x \in \mathbb{R}^{m},\right.
$$

with the penalty parameter $\eta>0$, and $f_{\eta}$ is the barrier function defined by

$$
f_{\eta}(x)= \begin{cases}b^{T} x+n \eta \ln \eta-\eta \sum_{i=1}^{n} \ln \left\langle e_{i}, A^{T} x-c\right\rangle & \text { if } A^{T} x-c>0 \\ +\infty & \text { if not }\end{cases}
$$

where $\left(e_{1}, e_{2}, \ldots, e_{n}\right)$ is the canonical base in $\mathbb{R}^{n}$. We are interested then in solving the prob$\operatorname{lem}\left(D_{\eta}\right)$.

The idea of this new approach consists to introduce one original process to calculate the step-size based on minorant functions.

The main advantage of $\left(D_{\eta}\right)$ resides in the strict convexity of its objective function and its feasible domain. Consequently, the conditions of optimality are necessary and sufficient. This fosters theoretical and numerical studies of the problem.

We study in the next section, the existence and uniqueness of optimal solution of the problem $\left(D_{\eta}\right)$, and we show its convergence to problem $(D)$, in particular the behavior of its optimal value and its optimal solutions when $\eta \rightarrow 0$, then $\lim _{\eta \rightarrow 0} x_{\eta}=x^{*}$ is an optimal solution of $(D)$. 
In Section 3, we propose an interior point algorithm based on the Newton's approach which allows us to solve the nonlinear system resulting from the optimality conditions. The iteration of this algorithm is of descent type, defined by $x_{k+1}=x_{k}+\alpha_{k} d_{k}$, where $d_{k}$ is the descent direction and $\alpha_{k}$ is the step-size. Also, we present different steps-size by minimizing a minorant functions which approximate the unidimensional function $\theta\left(\alpha_{k}\right)=\min _{\alpha>0} f(x+\alpha d)$. The last section, is dedicated to the presentation of comparative numerical tests to illustrate the effectiveness of our approaches and to determine the most efficient algorithm.

The main advantage of $\left(D_{\eta}\right)$ resides in the strict convexity of its objective function and its feasible domain. Consequently, the conditions of optimality are necessary and sufficient. This, fosters theoretical and numerical studies of the problem.

Before this, it is necessary to show that $\left(D_{\eta}\right)$ has at least an optimal solution.

\section{Existence and uniqueness of optimal solution of perturbed problem and its convergence to problem $(D)$}

\subsection{Existence and uniqueness of optimal solution of perturbed problem}

Firstly, we give the following definition

Definition 1.1. Let $f$ be a function defined from $\mathbb{R}^{m}$ to $\mathbb{R} \cup\{\infty\}, f$ is called inf-compact if for all $\eta>0$, the set $X_{\eta}(f)=\left\{x \in \mathbb{R}^{m}: f(x) \leqslant \eta\right\}$ is compact, which comes in particular to say that its cone of recession is reduced to zero.

To prove that $\left(D_{\eta}\right)$ has an optimal solution, we show that $f_{\eta}$ is inf-compact. For that, it is enough to prove that the cone of recession

$$
\widehat{X}\left(\left(f_{\eta}\right)_{\infty}\right)=\left\{d \in \mathbb{R}^{n},\left(f_{\eta}\right)_{\infty}(d) \leqslant 0\right\}
$$

is reduced to the origin, i.e., $\left(\left(f_{\eta}\right)_{\infty}(d) \leqslant 0\right) \Rightarrow(d=0)$, where $\left(f_{\eta}\right)_{\infty}$ is defined by

$$
\left(f_{\eta}\right)_{\infty}(d)=\lim _{\alpha \rightarrow+\infty} \frac{f_{\eta}(x+\alpha d)-f_{\eta}(x)}{\alpha}=b^{T} d .
$$

This needs to prove the following proposition.

Proposition $1([6])$. $d=0$ whenever $b^{T} d \leqslant 0$ and $A^{T} d \in \widehat{X}$.

Then, The problem $\left(D_{\eta}\right)$ has an optimal solution.

We know that the Hessian matrix $H=\nabla^{2} f_{\eta}(x)$ is positive definite, then the problem $\left(D_{\eta}\right)$ is strictly convex and if it has an optimal solution then it is unique.

We have

$$
f_{\eta}(x)=b^{T} x+n \eta \ln \eta-\eta \ln \sum_{i=1}^{n}\left\langle e_{i}, A^{T} x-c\right\rangle
$$

then

$$
\nabla f_{\eta}(x)=b-\eta \sum_{i=1}^{n} \frac{A e_{i}}{\left\langle e_{i}, A^{T} x-c\right\rangle}
$$

and

$$
\nabla^{2} f_{\eta}(x)=\eta \sum_{i=1}^{n} \frac{A e_{i}\left(A e_{i}\right)^{T}}{\left\langle e_{i}, A^{T} x-c\right\rangle^{2}} .
$$

As $f_{\eta}$ is inf-compact and strictly convex, therefore the problem $\left(D_{\eta}\right)$ admits a unique optimal solution.

We denote by $x(\eta)$ or $x_{\eta}$ the unique optimal solution of $\left(D_{\eta}\right)$. 


\subsection{Convergence of the perturbed problem to the problem $(D)$}

For $x \in \widehat{X}$, let's introduce the symmetrical definite positive matrix $B_{i}$ of rank $m, i=1, \ldots, n$ and the lower triangular matrix $L$, such that

$$
B_{i}=A e_{i}\left(A e_{i}\right)^{T}=L L^{T}
$$

which implies that $H$ is a positive definite matrix.

In what follows, we will be interested by the behavior of the optimal value and the optimal solution $x(\eta)$ of the problem $\left(D_{\eta}\right)$. For that, let us introduce the function $\theta$ defined by

$$
\theta(x, \eta)=f_{\eta}(x)=\left\{\begin{array}{ll}
f_{\eta}(x) & \text { if } \\
+\infty & \text { if not. }
\end{array} A^{T} x-c>0\right.
$$

Proposition 2 ([6]). For $\eta>0$, let $x_{\eta}$ an optimal solution of the problem $\left(D_{\eta}\right)$, then there exists $x \in X$ an optimal solution of $(D)$, such that, $\lim _{\eta \rightarrow 0} x_{\eta}=x$.

Remark 1. We know that if one of the problems $(D)$ and $(P)$ has an optimal solution, and the values of their objective functions are equal and finite, the other problem has an optimal solution.

\section{Computation of the Newton descent direction}

In this part, we are interested in the numerical solution of the problem $\left(D_{\eta}\right)$. Interior point methods of types logarithmic barrier are conceived for solving this problem type while being based on the optimality conditions which are necessary and sufficient, $x_{\eta}$ is an optimal solution of $\left(D_{\eta}\right)$ if it satisfies the following condition

$$
\nabla f_{\eta}\left(x_{\eta}\right)=0
$$

To solve (1), we use the Newton's approach which means to find at each iteration a vector $x_{\eta k}+d_{k}$ checking the following linear system

$$
H_{k} d_{k}=-\nabla f_{\eta}\left(x_{\eta k}\right)
$$

As $H_{k}=\nabla^{2} f_{\eta}\left(x_{\eta k}\right)$ is a symmetric positive definite matrix, the Cholesky methods and the conjugate gradient methods are the best convenient for solving the system (2).

To ensure the convergence of the algorithm towards an optimal solution $x^{*}$ of $\left(D_{\eta}\right)$, it should be made sure that all the iterate $x_{\eta k}+d_{k}$ remains strictly feasible. For that, we introduce a step-size $\alpha_{k}$ checking the condition $A^{T}\left(x_{\eta k}+\alpha_{k} d_{k}\right)-c>0$.

In the next section, we calculate the step-size for our new approach.

\section{Computation of the step-size with the minorant functions}

In the descent methods, the line search methods are known to compute the optimal stepsize $\alpha_{k}$. It suffice to minimize the unidimensional function $\theta\left(\alpha_{k}\right)=\min _{\alpha>0} f_{\eta}(x+\alpha d)$.

The most used methods of the type line search are those of Goldstein-Armijo, Fibonacci, etc. Unfortunately, these methods are expensive in computational volume, and even inapplicable to semidefinite problems. To avoid this difficulty, we exploit the idea suggested by J. P. Crouzeix and B. Merikhi [2] which approaches the function

$$
\varphi(\alpha)=\frac{1}{\eta}\left[f_{\eta}(x+\alpha d)-f_{\eta}(x)\right]
$$


by the simple majorant function giving at each iteration $k$, a step-size $\alpha_{k}$ in an easy way, simple and much less expensive than line search methods.

But, we propose a new idea, we suggest the simple minorant functions, we approaches the function (4).

Remark 2. To keep the function $\varphi(\alpha)$ well defined, it is necessary that for all $x \in \widehat{X},(x+\alpha d)$ still in $\widehat{X}$. Which returns to find $\widehat{\alpha}>0$ such that for any $\alpha \in[0, \widehat{\alpha}], x+\alpha d \in \widehat{X}$.

Proposition 3 ([6]). Let $\widehat{\alpha}=\sup \left\{\alpha, 1+z_{i} \alpha\right\}$ with $z_{i}=\frac{\left\langle e_{i}, A^{T} d\right\rangle}{\left\langle e_{i}, A^{T} x-c\right\rangle}, \forall i=1, \ldots, n$. Far all $\alpha \in[0, \widehat{\alpha}]$, the following function $\varphi(\alpha)$ is well defined

$$
\varphi(\alpha)=n\left(\sum_{i=1}^{n} z_{i}\right) \alpha-\|z\|^{2} \alpha-\sum_{i=1}^{n} \ln \left(1+z_{i} \alpha\right), \alpha \in[0, \widehat{\alpha}] .
$$

\subsection{Some useful inequalities}

Before determining these functions, we need the following results

The following result is caused by H. Wolkowicz and al. [9], see also J. P. Crouzeix and al. [3] for additional results.

Proposition 4 ( [9]).

$$
\begin{aligned}
& \bar{x}-\sigma_{x} \sqrt{n-1} \leqslant \min _{i} x_{i} \leqslant \bar{x}-\frac{\sigma_{x}}{\sqrt{n-1}}, \\
& \bar{x}+\frac{\sigma_{x}}{\sqrt{n-1}} \leqslant \max _{i} x_{i} \leqslant \bar{x}+\sigma_{x} \sqrt{n-1} .
\end{aligned}
$$

Let's recall that, B. Merikhi and al. (2008) [2] proposed some useful inequalities related to the maximum and to the minimum of $x_{i}>0$ for any $i=1, \ldots, n$

$$
n \ln \left(\bar{x}-\sigma_{x} \sqrt{n-1}\right) \leqslant A \leqslant \sum_{i=1}^{n} \ln \left(x_{i}\right) \leqslant B \leqslant n \ln (\bar{x}),
$$

with

$$
\begin{aligned}
A & =(n-1) \ln \left(\bar{x}+\frac{\sigma_{x}}{\sqrt{n-1}}\right)+\ln \left(\bar{x}-\sigma_{x} \sqrt{n-1}\right) \\
B & =\ln \left(\bar{x}+\sigma_{x} \sqrt{n-1}\right)+(n-1) \ln \left(\bar{x}-\frac{\sigma_{x}}{\sqrt{n-1}}\right)
\end{aligned}
$$

Such that $\bar{x}$ and $\sigma_{x}$ are respectively, the mean and the standard deviation of a statistical series $\left\{x_{1}, x_{2}, \ldots, x_{n}\right\}$ of $n$ real numbers. These quantities are defined as follows

$$
\bar{x}=\frac{1}{n} \sum_{i=1}^{n} x_{i} \quad \text { and } \quad \sigma_{x}^{2}=\frac{1}{n} \sum_{i=1}^{n} x_{i}^{2}-\bar{x}^{2}=\frac{1}{n} \sum_{i=1}^{n}\left(x_{i}-\bar{x}\right)^{2} .
$$

Based on this results, we give in the following, new notions of the non expensive minorant functions for $\varphi$, that offers some variable steps-size to every iteration with a simple technique.

Thanks to definite positivity results in linear algebra, we propose three different alternatives that offers some variable steps-size $\alpha$ to every iteration.

The efficient one to the other can be translated by numerical tests that we will present at the end of this work. 


\subsection{The minorant functions}

We seek a minorant function $\widehat{\varphi}$ of $\varphi$ on $\left[0, \widehat{\alpha}_{i}\right], i=1,2$ and 3 , such that

$$
\|z\|^{2}=n\left(\bar{z}^{2}+\sigma_{z}^{2}\right)=\widehat{\varphi}^{\prime \prime}(0)=-\widehat{\varphi}^{\prime}(0), \widehat{\varphi}(0)=0
$$

In the following, we take $x_{i}=1+\alpha z_{i}, x=1+\alpha z$ and $\sigma_{x}=\alpha \sigma_{z}$.

\section{First minorant function}

This strategy consists to minimize the minorant approximations $\widetilde{\varphi}$ of $\varphi$ over $[0, \widehat{\alpha}[$. To be efficient, this minorant approximation needs to be simple and sufficiently near $\varphi$. In our case, it requires $0=\widetilde{\varphi}(0),\|\lambda\|^{2}=\widetilde{\varphi}^{\prime \prime}(0)=-\widetilde{\varphi}^{\prime}(0)$.

By applying inequalities $(6)$, we give $\sum_{i=1}^{n} \ln \left(x_{i}\right) \leqslant B$.

Where

$$
\begin{gathered}
-\left(\alpha\|z\|^{2}+\sum_{i=1}^{n} \ln \left(1+z_{i} \alpha\right)\right) \geqslant-\left(B+\alpha\|z\|^{2}\right), \\
n \bar{z}-\left(\alpha\|z\|^{2}+\sum_{i=1}^{n} \ln \left(1+z_{i} \alpha\right)\right) \geqslant n \bar{z}-\left(B+\alpha\|z\|^{2}\right) .
\end{gathered}
$$

Thus the first minorant function can be defined as follows

$$
\begin{aligned}
\varphi_{1}(\alpha) & =n \bar{z}-\left(B+\alpha\|z\|^{2}\right) \\
\varphi_{1}(\alpha) & =\delta_{1} \alpha-(n-1) \ln \left(1+\beta_{1} \alpha\right)-\ln \left(1+\gamma_{1} \alpha\right)
\end{aligned}
$$

with $\delta_{1}=n \bar{z}-\|z\|^{2}, \beta_{1}=\bar{z}-\frac{\sigma_{z}}{\sqrt{n-1}}$ and $\gamma_{1}=\bar{z}+\sigma_{z} \sqrt{n-1}$.

The minorant function $\varphi_{1}$ is definite and convex on $\left[0, \widehat{\alpha}_{1}\right]$ and we have $\varphi_{1}(\alpha) \leqslant \varphi(\alpha)$, with $\varphi_{1}(0)=0$ and $\varphi_{1}^{\prime \prime}(0)=-\varphi_{1}^{\prime}(0)=\|z\|^{2}$.

This minimum is obtained in $\bar{\alpha}_{1}=\alpha_{\text {opt }}$, such that, $\varphi_{1}^{\prime}(\alpha)=0$.

We are then coming back to solve the second order following equation

$$
\alpha^{2}-2 b \alpha+c=0, \text { with } b=\frac{1}{2}\left(\frac{n}{\delta_{1}}-\frac{1}{\beta_{1}}-\frac{1}{\gamma_{1}}\right) \text { and } c=\frac{-\|\lambda\|^{2}}{\beta_{1} \gamma_{1} \delta_{1}} .
$$

The roots of this equation are of the type $\alpha=b \pm \sqrt{b^{2}-c}$. Let's take one root of the two roots that belong to $\left[0, \widehat{\alpha}_{1}[\right.$.

\section{Second minorant function}

One can also thought of simpler functions than $\varphi_{1}$ involving only one logarithm.

We consider functions of the following type

$$
\breve{\varphi}(\alpha)=\breve{\delta} \alpha-\breve{\gamma} \ln (1+\breve{\beta} \alpha), \quad \alpha \in[0, \breve{\alpha}[,
$$

where in order to fulfill the requirements

$$
\|\lambda\|^{2}=\breve{\gamma} \breve{\beta}^{2}=\breve{\gamma} \breve{\beta}-\breve{\delta}, \quad \breve{\alpha}=\sup [\alpha: 1+\alpha \breve{\beta}>0] .
$$

We can also think of another minorant function $\varphi_{2}$ better approximating $\varphi_{1}$ than $\varphi_{3}$, i.e.,

$$
\varphi_{3}(\alpha) \leqslant \varphi_{2}(\alpha) \leqslant \varphi_{1}(\alpha)
$$


such that $\beta_{2}=\beta_{1}=\bar{z}-\frac{\sigma_{z}}{\sqrt{n-1}}, \delta_{2}=\gamma_{2} \beta_{2}-\|z\|^{2}$ and we are looking for $\gamma_{2}=\frac{\|z\|^{2}}{\beta_{2}^{2}}$ which checks (6), which gives

$$
\begin{aligned}
\varphi_{2}(\alpha) & =\delta_{2} \alpha-\gamma_{2} \ln \left(1+\beta_{2} \alpha\right), \\
\varphi_{2}(\alpha) & =\left(\frac{\|z\|^{2}}{\beta_{2}}-\|z\|^{2}\right) \alpha-\frac{\|z\|^{2}}{\beta_{2}^{2}} \ln \left(1+\beta_{2} \alpha\right) .
\end{aligned}
$$

\section{Third minorant function}

Another minorant function simpler than $\varphi_{1}$ to extract from the known inequality

$$
\left(\|z\|-\sum_{i=1}^{n} z_{i}\right) \alpha-\sum_{i=1}^{n} \ln \left(1+z_{i} \alpha\right)-\ln (1+\|z\| \alpha) \leqslant 0
$$

where

$$
\varphi_{3}(\alpha)=\delta_{3} \alpha-\ln \left(1+\beta_{3} \alpha\right), \quad \alpha \in\left[0, \widehat{\alpha}_{3}\right],
$$

with $\widehat{\alpha}_{3}=\frac{-1}{\beta_{3}}, \delta_{3}=-\|\lambda\|(\|\lambda\|-1)$ and $\beta_{3}=\|\lambda\|$.

The minorant function $\varphi_{2}$ is definite and convex on $\left[0, \widehat{\alpha}_{3}\right]$ and we have $\varphi(\alpha) \geqslant \varphi_{3}(\alpha)$, with $\varphi_{3}(0)=0$ and $\varphi_{3}^{\prime \prime}(0)=-\varphi_{3}^{\prime}(0)=\|z\|^{2}$.

Proposition 5. $\varphi_{i}, i=1,2,3$, is strictly convex over $\left[0, \stackrel{\circ}{\alpha}\left[\right.\right.$, with $\stackrel{\circ}{\alpha}=\min \left(\widehat{\alpha}, \widehat{\alpha}_{1}, \widehat{\alpha}_{2}\right), \varphi(\alpha) \rightarrow$ $+\infty$ when $\alpha \rightarrow \widehat{\alpha}$. So we have

$$
\varphi_{3}(\alpha) \leqslant \varphi_{2}(\alpha) \leqslant \varphi_{1}(\alpha) \leqslant \varphi(\alpha), \forall \alpha \in[0, \stackrel{\circ}{\alpha} .
$$

Proof. The first inequality is obvious. The inequality $\varphi(\alpha) \geqslant \varphi_{1}(\alpha)$ is a direct consequence of (6). Let's consider, $g(\alpha)=\varphi_{2}(\alpha)-\varphi_{1}(\alpha)$. Since $\beta_{1}=\beta_{2}$ and $\beta_{1} \leqslant \gamma_{1}$, we have for any $\alpha \in[0, \stackrel{\alpha}{\alpha}[$

$$
g^{\prime \prime}(\alpha)=\frac{\gamma_{2} \beta_{2}^{2}-(n-1) \beta_{1}^{2}}{\left(1+\beta_{1} \alpha\right)^{2}}-\frac{\gamma_{1}^{2}}{\left(1+\gamma_{1} \alpha\right)^{2}} \leqslant \frac{\gamma_{1}^{2}}{\left(1+\beta_{1} \alpha\right)^{2}}-\frac{\gamma_{1}^{2}}{\left(1+\gamma_{1} \alpha\right)^{2}} \leqslant 0,
$$

and since $g(0)=g^{\prime}(0)=0$ and $g^{\prime \prime}(\alpha) \leqslant 0$, it becomes $g(\alpha) \leqslant 0$ for any $\alpha \in[0, \propto[$.

Then, let's put $h(\alpha)=\varphi_{3}(\alpha)-\varphi_{2}(\alpha)$, so

$$
h(0)=h^{\prime}(0)=0 \quad \text { and } \quad h^{\prime \prime}(\alpha)=\frac{\beta_{3}^{2}}{\left(1+\beta_{3} \alpha\right)^{2}}-\frac{\gamma_{2} \beta_{2}^{2}}{\left(1+\beta_{2} \alpha\right)^{2}} .
$$

Since $\|z\|^{2}=\gamma_{2} \beta_{2}^{2}$ and so $\beta_{3}=\|z\|$

$$
h^{\prime \prime}(\alpha)=\|z\|^{2}\left(\frac{1}{\left(1+\beta_{3} \alpha\right)^{2}}-\frac{1}{\left(1+\beta_{2} \alpha\right)^{2}}\right) \leqslant 0
$$

because $\beta_{2} \leqslant \beta_{3}$.

Therefore $h(\alpha) \leqslant 0$ for any $\alpha \in[0, \stackrel{\circ}{\alpha}[$.

Thus, we deduce that the function $\varphi_{i}$ reaches its minimum at a unique point $\bar{\alpha}_{i}$ which is the root of $G_{i}^{\prime}(\alpha)=0$. Thus, the three roots are explicitly calculated, for $i=1,2,3$, we have

$$
\begin{aligned}
\bar{\alpha}_{1} & =b-\sqrt{b^{2}-c} \text { with } b=\frac{1}{2}\left(\frac{n}{\delta_{1}}-\frac{1}{\beta_{1}}-\frac{1}{\gamma_{1}}\right) \text { and } c=\frac{-\|z\|^{2}}{\beta_{1} \gamma_{1} \delta_{1}}, \\
\bar{\alpha}_{2} & =\frac{\gamma_{2}}{\delta_{2}}-\frac{1}{\beta_{2}} \\
\bar{\alpha}_{3} & =\frac{-1}{\|z\|-1} .
\end{aligned}
$$


For $\bar{\alpha}_{1}$, we take the root that belongs to the interval $(0, \widehat{\alpha})$.

Thus, the three values $\bar{\alpha}_{i}, i=1,2,3$ are explicitly computed, then, we take $\bar{\alpha}_{1}, \bar{\alpha}_{2}$ and $\bar{\alpha}_{3}$ are belongs to the interval $(0, \widehat{\alpha}-\varepsilon)$ and $\varphi^{\prime}(\alpha)<0$, with $\varepsilon>0$, is a fixed precision.

Remark 3. The calculation of $\bar{\alpha}$ is performed by a dichotomous procedure, in the cases where $\bar{\alpha}_{i} \notin(0, \widehat{\alpha}-\varepsilon)$, and $\varphi^{\prime}(\alpha)>0$, as follows

$$
\begin{aligned}
& \text { put } a=0 \text { and } b=\widehat{\alpha}-\varepsilon \\
& \text { while }|b-a|>10^{-4} \\
& \text { if } \varphi\left(\frac{a+b}{2}\right)<0 \text { then } b=\frac{a+b}{2} \\
& \text { else } a=\frac{a+b}{2}, \text { so } \bar{\alpha}=b .
\end{aligned}
$$

This calculation guarantees a better approximation of the minimizer of $\varphi^{\prime}(\alpha)$ while remaining in the domain of $\varphi$.

Proposition $6([6])$. Let $x_{k+1}$ and $x_{k}$ two strictly feasible solutions of $\left(D_{\eta}\right)$, obtained respectively at the iteration $k+1$ and $k$, so we have $f_{\eta}\left(x_{k+1}\right)<f_{\eta}\left(x_{k}\right)$.

\section{The algorithm}

In this section, we present the algorithm of our approach to obtain an optimal solution $\bar{x}$ to the problem $(D)$.

For simplicity, we consider $x_{k}$ instead of $x_{\eta k}$ and $x$ instead of $x_{\eta}$.

\section{Begin algorithm}

\section{Initialization}

$x_{0}$ is a strictly feasible solution of $(D), d_{0} \in \mathbb{R}^{m}, \varepsilon>0$ is a given precision.

\section{Iteration}

- While $\left|b^{T} d^{k}\right|>\varepsilon$ do

1. Solve the system $H_{k} d_{k}=-\nabla f_{\eta}\left(x_{k}\right)$.

2. Compute the step-size using the strategies $S_{i}, i=1,2,3$.

3. Take the new iterate $x^{k+1}=x^{k}+\alpha_{k} d_{k}$.

4. Take $k=k+1$.

\section{- End while}

\section{End algorithm}

This approach reaches to reduce the number of the iteration and the time of calculation. In the following Section, we present some examples.

\section{Numerical tests}

The following examples are taken from the literature see for instance $[1,4,8]$ and implemented in MATLAB R2013a on Pentium(R) Dual Core CPU T4400 (2.20 GHz) with 3.00 Go RAM. We have taken $\varepsilon=1.0 e-006$. In the table of results, (size) represents the size of the example, (itrat) represents the number of iterations necessary to obtain an optimal solution, (time) represents the time of computation in seconds (s) and (st) represents the strategy.

We note that the matrices used in the numerical tests are full matrices. 


\section{Examples of fixed sizes}

Example 01:

$$
A=\left[\begin{array}{ccc}
1 & -1 & 0 \\
1 & 1 & 1
\end{array}\right], b=\left[\begin{array}{l}
0 \\
1
\end{array}\right] \text { and } c=\left[\begin{array}{lll}
1 & 1 & 0
\end{array}\right]^{t}
$$

Example 02:

$$
A=\left[\begin{array}{cccc}
2 & 3 & 1 & 2 \\
3 & 0 & -2 & 1
\end{array}\right], b=\left[\begin{array}{l}
2 \\
0
\end{array}\right] \text { and } c=\left[\begin{array}{cccc}
4 & 1 & 2 & 0
\end{array}\right]^{t}
$$

Example 03:

$$
A=\left[\begin{array}{cccc}
1 & -1 & 1 & 1 \\
2 & 1 & -1 & 2 \\
1 & 1 & 1 & 2
\end{array}\right], b=\left[\begin{array}{l}
3 \\
4 \\
5
\end{array}\right] \text { and } c=\left[\begin{array}{llll}
3 & 2 & 1 & 3
\end{array}\right]^{t}
$$

Example 04:

$$
A=\left[\begin{array}{cccccc}
2 & 1 & 0 & -1 & 0 & 0 \\
0 & 0 & 1 & 0 & 1 & -1 \\
1 & 1 & 1 & 1 & 1 & 1
\end{array}\right], b=\left[\begin{array}{l}
0 \\
0 \\
1
\end{array}\right] \text { and } c=\left[\begin{array}{cccccc}
3 & -1 & 1 & 0 & 0 & 0
\end{array}\right]^{t} .
$$

Example 05:

$$
A=\left[\begin{array}{ccccccc}
-1 & 1 & 1 & -1 & 1 & 0 & 0 \\
0 & 2 & -3 & 2 & 0 & 1 & 0 \\
-3 & 2 & 1 & 0 & 0 & 0 & 1 \\
3 & 5 & 4 & 0.5 & 0 & 0 & 0
\end{array}\right], b=\left[\begin{array}{l}
1 \\
2 \\
0 \\
2
\end{array}\right] \text { and } c=\left[\begin{array}{lllllll}
1 & 1 & 0 & 0 & 1 & 1 & -2
\end{array}\right]^{t}
$$

Example 06:

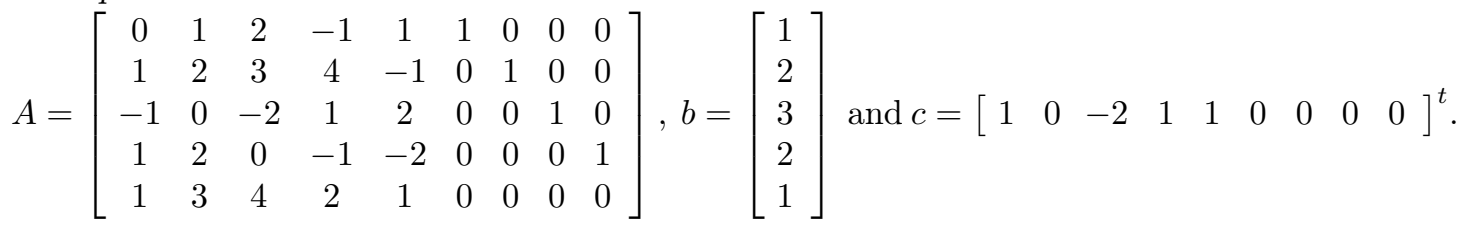

Example 07:

$$
\begin{aligned}
A & =\left[\begin{array}{cccccccccccc}
1 & 0 & -4 & 3 & 1 & 1 & 1 & 0 & 0 & 0 & 0 & 0 \\
5 & 3 & 1 & 0 & -1 & 3 & 0 & 1 & 0 & 0 & 0 & 0 \\
4 & 5 & -3 & 3 & -4 & 1 & 0 & 0 & 1 & 0 & 0 & 0 \\
0 & -1 & 0 & 2 & 1 & -5 & 0 & 0 & 0 & 1 & 0 & 0 \\
-2 & 1 & 1 & 1 & 2 & 2 & 0 & 0 & 0 & 0 & 1 & 0 \\
2 & -3 & 2 & -1 & 4 & 5 & 0 & 0 & 0 & 0 & 0 & 1
\end{array}\right], \quad b=\left[\begin{array}{c}
1 \\
4 \\
4 \\
5 \\
7 \\
5
\end{array}\right] \\
\text { and } c & =\left[\begin{array}{cccccccccccc}
-4 & -5 & -1 & -3 & 5 & -8 & 0 & 0 & 0 & 0 & 0 & 0
\end{array}\right]^{t} .
\end{aligned}
$$

Example 08:

The matrix $A$ is

$$
\left[\begin{array}{rrrrrrrrrrrrrrrrrrrrrrrrr}
1 & 1 & 0 & 0 & 0 & 0 & 0 & 0 & 0 & 0 & 0 & 0 & 0 & 0 & 1 & 0 & 0 & 0 & 0 & 0 & 0 & 0 & 0 & 0 & 0 \\
0 & 2 & 1 & 0 & 0 & 0 & 0 & 0 & 0 & 0 & 0 & 0 & 0 & 0 & 0 & 1 & 0 & 0 & 0 & 0 & 0 & 0 & 0 & 0 & 0 \\
0 & 0 & 3 & -1 & 0 & 0 & 0 & 0 & 0 & 0 & 0 & 0 & 0 & 0 & 0 & 0 & 1 & 0 & 0 & 0 & 0 & 0 & 0 & 0 & 0 \\
0 & 0 & 0 & 2 & 4 & 0 & 0 & 0 & 0 & 0 & 0 & 0 & 0 & 0 & 0 & 0 & 0 & 1 & 0 & 0 & 0 & 0 & 0 & 0 & 0 \\
0 & 0 & 0 & 0 & 6 & 2 & 0 & 0 & 0 & 0 & 0 & 0 & 0 & 0 & 0 & 0 & 0 & 0 & 1 & 0 & 0 & 0 & 0 & 0 & 0 \\
0 & 0 & 0 & 0 & 0 & -1 & 2 & 0 & 0 & 0 & 0 & 0 & 0 & 0 & 0 & 0 & 0 & 0 & 0 & 1 & 0 & 0 & 0 & 0 & 0 \\
0 & 0 & 0 & 0 & 0 & 0 & 4 & -1 & 0 & 0 & 0 & 0 & 0 & 0 & 0 & 0 & 0 & 0 & 0 & 0 & 1 & 0 & 0 & 0 & 0 \\
0 & 0 & 0 & 0 & 0 & 0 & 0 & 0 & 3 & 1 & 0 & 0 & 0 & 0 & 0 & 0 & 0 & 0 & 0 & 0 & 0 & 0 & 1 & 0 & 0 \\
0 & 0 & 0 & 0 & 0 & 0 & 0 & 0 & 3 & -1 & 1 & 0 & 0 & 0 & 0 & 0 & 0 & 0 & 0 & 0 & 0 & 0 & 1 & 0 & 0 \\
0 & 0 & 0 & 0 & 0 & 0 & 0 & 0 & 0 & 1 & 1 & 2 & 0 & 0 & 0 & 0 & 0 & 0 & 0 & 0 & 0 & 0 & 0 & 1 & 0 \\
0 & 0 & 0 & 0 & 0 & 0 & 0 & 0 & 0 & 0 & 0 & 1 & 2 & 1 & 0 & 0 & 0 & 0 & 0 & 0 & 0 & 0 & 0 & 0 & 1
\end{array}\right],
$$


the vector $c$ and $b$ are

$$
\begin{aligned}
c & =\left[\begin{array}{lllllllllllllllllllllllll}
2 & -1 & -3 & 5 & -2 & 0 & 4 & 1 & 2 & -1 & 1 & -1 & 0 & 2 & 0 & 0 & 0 & 0 & 0 & 0 & 0 & 0 & 0 & 0 & 0
\end{array}\right]^{t}, \\
b & =\left[\begin{array}{lllllllllll}
8 & 4 & 6 & 2 & 5 & 1 & 2 & 6 & 3 & 9 & 4
\end{array}\right]^{t} .
\end{aligned}
$$

\begin{tabular}{|c|c|c|c|c|c|c|}
\hline \multirow[t]{2}{*}{ size } & \multicolumn{2}{|l|}{$\mathrm{st}_{1}$} & \multicolumn{2}{|l|}{$\mathrm{st}_{2}$} & \multicolumn{2}{|l|}{$\mathrm{st}_{3}$} \\
\hline & itrat & time & itrat & time & itrat & time \\
\hline $2 \times 3$ & 2 & 0.0016 & 2 & 0.0016 & 4 & 0.0025 \\
\hline $2 \times 4$ & 5 & 0.032 & 5 & 0.032 & 7 & 0.045 \\
\hline $3 \times 4$ & 1 & 0.001 & 1 & 0.0019 & 4 & 0.0036 \\
\hline $3 \times 6$ & 6 & 0.044 & 6 & 0.042 & 9 & 0.076 \\
\hline $4 \times 7$ & 9 & 0.045 & 9 & 0.045 & 10 & 0.063 \\
\hline $5 \times 9$ & 8 & 0.049 & 8 & 0.049 & 12 & 0.085 \\
\hline $6 \times 12$ & 9 & 0.055 & 9 & 0.032 & 13 & 0.090 \\
\hline $11 \times 25$ & 13 & 0.048 & 13 & 0.055 & 15 & 0.099 \\
\hline
\end{tabular}

The last examples can be given in the following table

\section{Example cube}

$n=2 m, A[i, j]=0$ if $i \neq j$ or $(i+1) \neq j$.

$A[i, j]=A[i, i+m]=1, b[i]=2$, for $i, j=1, \ldots, m$.

\begin{tabular}{|c|c|c|c|c|c|c|}
\hline \multirow[t]{2}{*}{ size } & \multicolumn{2}{|l|}{$\mathrm{st}_{1}$} & \multicolumn{2}{|l|}{$\mathrm{st}_{2}$} & \multicolumn{2}{|l|}{$\mathrm{st}_{3}$} \\
\hline & itrat & time & itrat & time & itrat & time \\
\hline $50 \times 100$ & 1 & 0.031 & 4 & 0.096 & 9 & 0.25 \\
\hline $100 \times 200$ & 1 & 0.053 & 5 & 0.10 & 11 & 0.45 \\
\hline $200 \times 400$ & 2 & 0.088 & 5 & 0.29 & 14 & 0.555 \\
\hline $450 \times 900$ & 3 & 0.096 & 6 & 0.55 & 19 & 0.99 \\
\hline
\end{tabular}

The following table resumes the obtained results

\section{Commentary}

These tests show, clearly, the impact of our three strategies offer an optimal solution of $(D)$ and $(P)$ in a reasonable time and with a small number of iterations.

We notice that the 1st strategy is the best. The obtained comparative numerical results favor this last strategy moreover, it requires a computing time largely low vis-a-vis the other two strategies. This seems to be quite expected, because theoretically the strategy st ${ }_{1}$ uses the function $\varphi_{1}$ that is the closest (best approximation) of the function $\varphi$.

\section{Conclusion}

In spite of the mathematical development in the domain of the linear programming, a lot of problems remain to develop. For it, in our survey, we treated a theoretical and numerical survey of our new approach, based on the notion of majorant functions. This allows us to determine the displacement step by a simple and easy manner.

The numerical simulations confirm the effectiveness of our approaches. Our algorithm converges to the same optimal solution, using any strategy among the three proposed strategies. The first strategy is the best approach versus computing time and number of iterations.

Thus, the numerical tests prove that our approache was reducing the cost of iteration for the linear programming. Our survey, opens interesting perspective for the non linear programming (PNL). 


\section{References}

[1] J.F.Bonnans, J.C.Gilbert, C.Lemaréchal, C. Sagastizabal, Numerical optimization, theoritical and pratical aspects. Springer-Verlag, 2003.

[2] J.P.Crouzeix, B.Merikhi, A logarithm barrier method for semidefinite programming, RAIRO Oper. Res., 42(2008), 123-139.

[3] J.P.Crouzeix, A.Seeger, New bounds for the extreme values of a finite sample of real numbers, Journal of Mathematical Analysis and Applications, 197(1996), 411-426.

[4] R.M.Freund, S.Mizuno, Interior point methods: Current status and future directions, Mathematical Programming, Optima, no. 51, 1996.

[5] A.Leulmi, B.Merikhi, D.Benterki, Study of a Logarithmic Barrier Approach for Linear Semidefinite Programming, Journal of Siberian Federal University. Mathematics \& Physics, bf 11(2018), no. 3, 1-13.

[6] L.Menniche, Dj.Benterki, A logarithmic barrier approach for linear programming, Journal of Computational and Applied Mathematics, 312(2017), 267-275.

[7] R.T.Rockafellar, Convex analysis, Princeton University Press, New Jerzy, 1970.

[8] G.Savard, Introduction au méthodes de point intérieur, Extrait denotes, Département de Mathématiques et Génie Industriel, Ecole Polytechnique de Montréal, Fevrier, 2001.

[9] H.Wolkowicz, G.P.H.Styan, Bounds for eigenvalues using traces, Linear Algebra and Appl., 29(1980), 471-506.

\section{Метод логарифмического барьера через минорантную функцию для линейного программирования}

Ассма Леулми

Кафедра математики, факультет наук Ферхат Аббас Университет Сетиф-1, 19000

Алжир

Соумия Леулми

Кафедра математики

Университет Мохамеда Хидера в Бискре

Алжир

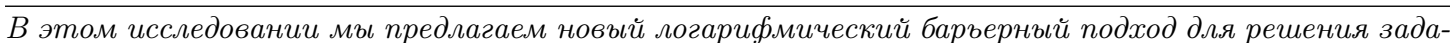
чи линейного программирования. Мы заинтересованы в вычислении направления по методу Нъютона и шага смещения с использованием функций миноранта вместо методов поиска строк, чтобы уменъшить стоимость вычислений.

Наш новый подход еще более полезен, чем классические методы линейного поиска. Он подтверждается многими интересными численными экспериментами, показавшими эфбективность алгоритма, разработанного в данной работе.

Ключевые слова: линейное программирование, метод логарифмического баръера, поиск линии. 